\title{
Brain Tumor Analysis in Basf Framework
}

\author{
P. Kavitha, S. Prabakaran
}

\begin{abstract}
The medical image processing is extensively used in several areas. In earlier detection and treatment of these diseases is very helpful to find out the abnormality issues in that image. Here there are number of methods available for diagnosis to detect the brain tumor of MRI image. This study deals with there are two main contributions are implemented in this filter method. (1)The extension of adaptive bilateral method to apply sub-bands of low frequency signal decomposed using wavelet transform. A wavelet threshold is combined with adaptive bilateral method to form an innovative structure in image de-noising method. It's very efficient to eliminate noise in original noisy images. (2) First detected block boundary and texture regions discontinuities to adapt or control the parameters of spatial and intensity in bilateral filter. The adaptive method can improve the restored image quality in this test result compared with standard bilateral filter. The proposed segmentation technique uses novel strip method and the image is split into number of strips 3, 4, 5 and 6. Using a hybrid Assured Convergence PSO (ACPSO) and Fuzzy C-Mean Clustering (FCM) was proposed method. The segmentation algorithm presented in this research gives $95 \%$ of accuracy rate to detect brain tumor when strip count is 5 . In this research work presented a feature vector using a different statistical texture analysis of brain tumor from MRI image. The statistical feature texture is computed using GLCM (Gray Level Co-occurrence Matrices) of Brain Nodule structure. For this research work, the brain nodule segmented using strips method to implemented marker watershed image segmentation based on PSO (Particle Swarm Optimization) and Fuzzy C-means clustering (FCM). Furthermore, the four angles $00,450,900$ and 1350 are calculated the segmented brain image in GLCM. The four angular directions are calculated using texture features are correlation, energy, contrast and homogeneity. The accuracy rate of previous method was compared and proved the proposed method an Assured Convergence Particle Swarm Optimization (ACPSO)-Fuzzy C-Mean (FCM) and using SVM classification technique is suitable for the early detection of brain tumor. In proposed, a tumor extraction is improved in ASPSO-FCM and SVM classification with better accuracy rate of $95.31 \%$.
\end{abstract}

Keywords: Bilateral, PSO, FCM, GLCM, SVM

\section{INTRODUCTION}

The medical image processing is extensively used in several areas. In earlier detection and treatment of these diseases is very helpful to find out the abnormality issues in that image. In this study, a diagnosis process of brain tumor with help of MRI scan images. In earlier detection and

Revised Manuscript Received on July 22, 2019.

P. Kavitha, Department of Information Technology, Bharath Institute of Higher Education and Research, Chennai, India.

Email: kavithaprithiviraj2019@yahoo.com

Dr. S. Prabakaran, Department of Computer Science and Engineering, SRM University, Kattankulathur, Chennai, India,

E-mail:prabakaran.s@srmuniv.ac.in treatment of these diseases is very helpful to find out the abnormality issues in that image. Here there are number of methods available for segmentation to detect the brain tumor of MRI scan image. The proposed technique of this study, the earlier detection of brain tissues using different techniques has been introduced for image de-noising and segmenting. This study proposed an efficiency model in which an image de-noising using the method of multi-resolution bilateral filter technique and the proposed segmentation technique uses novel strip method and the image is split into number of strips 3, 4, 5 and 6. A hybrid Assured Convergence PSO (ACPSO) and Fuzzy C-Mean Clustering (FCM) was proposed method. In segmentation algorithm presented in this paper gives $95 \%$ of accuracy rate to detect brain tissues when strip count is 4 . The combination of these two proposed techniques is giving better accuracy rate in least computational period.

\section{LITERATURE SURVEY}

Emre Dandl [1] was proposed method to classify the lung nodules. In LUVEM (Lung Volume Extraction Method), using median filter to reduce the noise and to implement LUVEM method to extract the nodules from CT scan image. After that the Self Organizing Method (SOM) is successful detection of early stages. It's unsupervised neural network method and easily segmented to small nodules on the lung nodules. In feature extraction, first we used shape based method for analyzing lung nodule image and to utilize GLCM method is used to statistic of gray level lung nodule. Finally the lung nodules are extracted and computed to the segmentation object. Finally, using Probabilistic Neural Network (PNN) to classify the bengin and malign lung nodule. The result $f$ the proposed classification method of PNN is given better accuracy value.

Lavanya M, Muthu Kannan P was proposed comparative analysis of FCM and FLICM algorithm. Pre-processing the input image to reduce noise reduction and histogram equalization is done using median filer and filtering image is using segmentation of Modified Expectation Maximization (MEM) algorithm. This algorithm to initialize the parameter of expectation and maximization and the value is increased step by step. Finally, calculate the new maximization and expectation position. After that the completion of segmentation to extract the nodule is utilized for feature extraction method such area, perimeter and eccentricity. Final steps to classification method to compared better best performance measures of FCM and FLICM. The performance measures of specificity $92 \%$, precision $97 \%$, sensitivity $82 \%$ and accuracy $95 \%$ [2]. 
The author is mainly focused to implement soft computing methods. In pre-processing, various filtering methods median, gabor filter and anisotropic filtering are compared and proved anisotropic filter are more suitable for pre-processing. A Marker based Watershed algorithm is used to segment the lung nodule to extract the image. Finally, compared SVM and KNN classification result the KNN classifier to provide better accuracy value of the image and this proposed method was implemented by Shraddha G. Kulkarni, Sahebrao B. Bagal [3].

\section{PROPOSED TECHNIQUES}

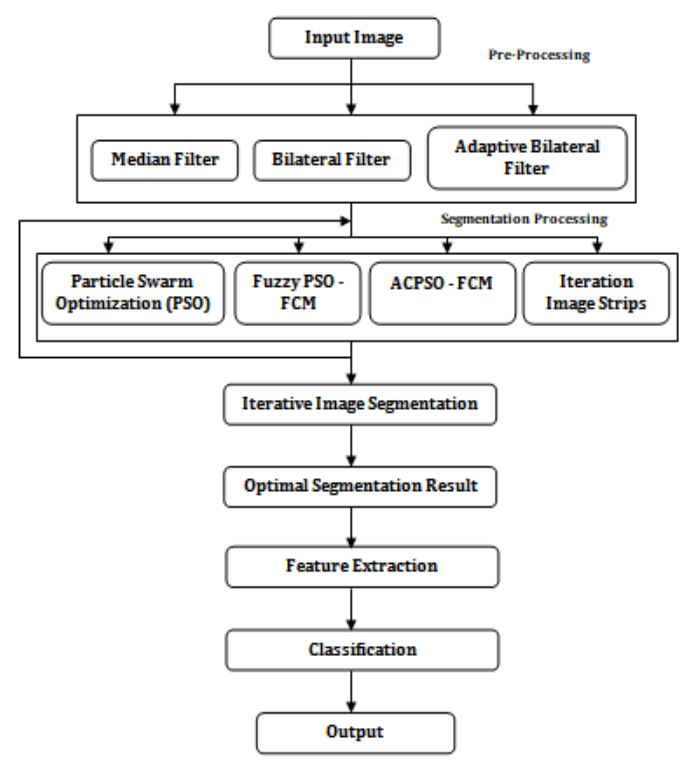

Figure 1: Block Diagram of Research Methodology

\section{A. Pre-Processing}

I propose a two main contribution are implemented in this pre-processing technique:

(1) The extension of bilateral filter to enhance multi-resolution of adaptive bilateral filter, where the bilateral filter is applied to low frequency sub-bands of a signal. A signal is decomposed using wavelet transform filter. A multi-resolution adaptive bilateral filter is combined with wavelet transform threshold. It's a new framework to reduce noisy image, very efficient to remove the noise in original noisy image.

(2) To remove compression artifacts using spatial adaptive method. This method to avoid in excess of smoothing image texture regions and to effectively remove the blocking artifacts and ringing artifacts, in this research, first detected block boundary and texture regions used to adapt/control the Gaussian filters of spatial domain and intensity domain parameters of the bilateral filter. The experimental results prove the adaptive method can improve the image quality is better than the standard bilateral filter.

\section{B. Segmentation}

The proposed segmentation technique uses novel strip method and the image is split into number of strips 3, 4, 5 and

6. A hybrid Assured Convergence PSO (ACPSO) and Fuzzy C-Mean Clustering (FCM) was proposed method. In segmentation algorithm presented in this paper gives $95 \%$ of accuracy rate to detect brain tissues when strip count is 4 .

\section{Feature Extraction}

The sample images analyzed texture features correlation, energy, contrast and homogeneity. In an Image, it measures the local intensity value changes in contrast features. If the image intensity contains even the contrast value is zero. If the contrast value is high the image intensity value also increased. The correlation ranges from -1 to 1 and the correlation measures hoe the image pixel is correlated to other neighbour image pixels. The similarity of image pixel is measured using homogeneity. The maximum count of the pixel is similar intensity means the homogeneity is high. The minimum intensity changes are occurred in the textures. The energy is also known as ASM (Angular Second Moment) it performs uniform intensity. The energy rate is 1 in the constant intensity image. Furthermore, the four angles 0o, 45o, 90o and 135o are calculated the segmented brain image in GLCM. The four angular directions are calculated using texture features are correlation, energy, contrast and homogeneity. The texture analysis is performed a different types of images using past years. So the algorithm proposed statistical texture features are calculated for iterative image segmentation. These results show that MRI image can be implemented in a system of brain cancer detection.

\section{Classification}

The classification technique is divided into two categories: training data part and testing part. In training part, the collection of data are given (i.e. 4 features $* 30$ images) to the classifier for training. In testing part, the collection of training data are given to the classification technique and the classification are perform better accuracy value and better error value of the classification. This accuracy and error value of the classification method is depends on the better efficiency of the training.

\section{EXPERIMENT RESULT AND DISCUSSION}

Based on this experiments results were discussed with previous methods, using parameters of bilateral method: · d $=1.8, \cdot \mathrm{r}=2^{*} \cdot \mathrm{n}$, and the image size $11 \mathrm{x} 11$. The previous methods were compared with proposed technique adaptive bilateral filter. In this proposed, to set the parameter value $\mathrm{d}=1.8, \cdot \mathrm{r}=1.0^{*} \cdot \mathrm{n}$, and the image size is $7 \times 7$ at each level.

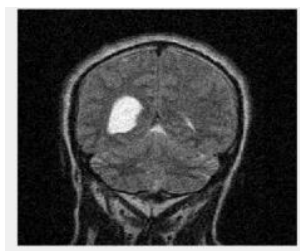

(a)

(b)
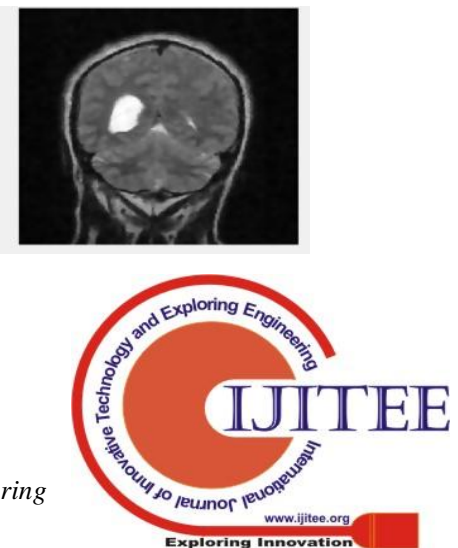
Figure 2: (a) Input Image (b) Proposed method, $\sigma_{\mathrm{d}}=1.8, \sigma_{\mathrm{r}}=1.0$ and $\sigma_{\mathrm{n}}$ at each level

The MSE and PSNR values were compared with previous methods the proposed adaptive bilateral method was proved better result.

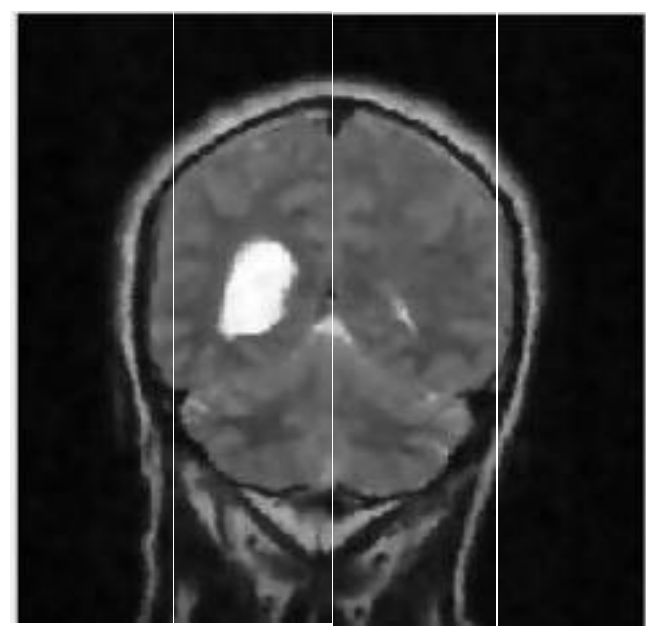

Figure 3: The filter image is subdivided into five horizontal equidistance strips

In this proposed method, the filter image is subdivided into five horizontal equivalent strips

The strips are numbered str1, str2, str3 and str4. The strip str2 is considered for segmentation.

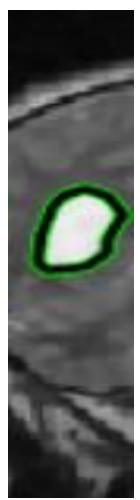

(a)

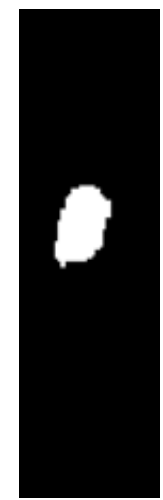

(b)
Figure 5: (a) The strip str2 is considered for segmentation (b) Image Extract

Table1. A comparison of PSNR value using various methods.

\begin{tabular}{|c|c|c|c|}
\hline $\begin{array}{c}\text { Sample } \\
\text { Image }\end{array}$ & $\begin{array}{c}\text { Media } \\
\mathbf{n}[\mathbf{5}]\end{array}$ & $\begin{array}{c}\text { Bilateral } \\
\text { [1] }\end{array}$ & Proposed \\
\hline Image_1 & 32.47 & 32.89 & 33.96 \\
\hline Image_2 & 32.97 & 33.41 & 33.53 \\
\hline
\end{tabular}
method classification with better accuracy rate of $95.31 \%$. In future, using different

\begin{tabular}{|c|c|c|c|c|c|}
\hline $\begin{array}{c}\text { Sample } \\
\text { Image }\end{array}$ & TPR & TNR & FPR & FNR & Accuracy \\
\hline Image_1 & $\begin{array}{c}87.541 \\
3\end{array}$ & 90.1196 & 9.8804 & $\begin{array}{c}12.458 \\
7\end{array}$ & 89.0624 \\
\hline Image_2 & $\begin{array}{c}85.961 \\
2\end{array}$ & 85.9612 & 8.5479 & 8.5479 & 88.9689 \\
\hline Image_3 & $\begin{array}{c}81.127 \\
1\end{array}$ & 88.7838 & $\begin{array}{c}11.216 \\
2\end{array}$ & $\begin{array}{c}18.872 \\
9\end{array}$ & 84.9967 \\
\hline Image_4 & $\begin{array}{c}79.138 \\
7\end{array}$ & 85.0084 & $\begin{array}{c}14.991 \\
6\end{array}$ & $\begin{array}{c}20.861 \\
3\end{array}$ & 81.8114 \\
\hline Image_5 & $\begin{array}{c}79.465 \\
5\end{array}$ & 85.157 & 14.843 & $\begin{array}{c}20.534 \\
5\end{array}$ & 82.0954 \\
\hline
\end{tabular}

Table 3: Comparative analysis of various algorithms and proposed

\begin{tabular}{|l|c|}
\hline \multicolumn{1}{|c|}{ Methods } & $\begin{array}{c}\text { Accuracy Rate } \\
(\boldsymbol{\%})\end{array}$ \\
\hline PSO, GA and SVM & 89.3 \\
\hline FPSO-FCM and SVM & 92.17 \\
\hline $\begin{array}{l}\text { Proposed ACPSO-FCM } \\
\text { and SVM }\end{array}$ & 95.31 \\
\hline
\end{tabular}

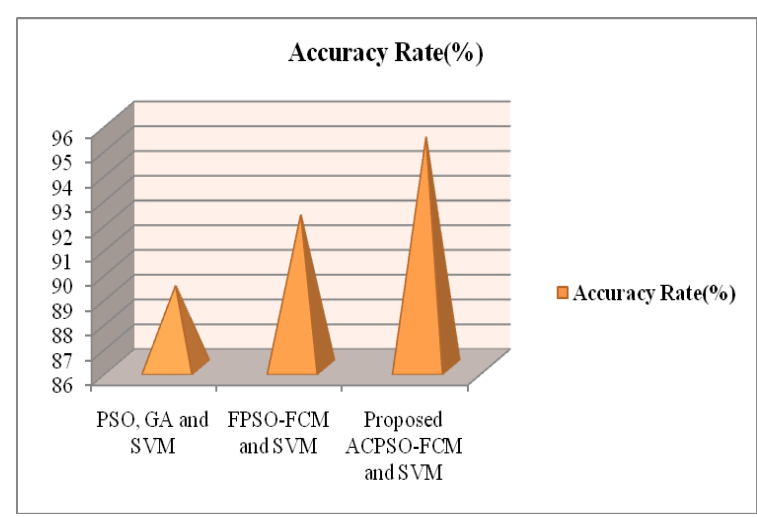

Figure 3: Graphical view analysis of proposed method

The accuracy rate of this technique $92.17 \%$ [13] and the graphical view analysis of previous techniques was compared and proved the proposed method an Assured Convergence Particle Swarm Optimization (ACPSO)-Fuzzy C-Mean (FCM) and using SVM classification technique is suitable for the early detection of brain tumor. In proposed, a tumor extraction is improved in ASPSO-FCM and SVM

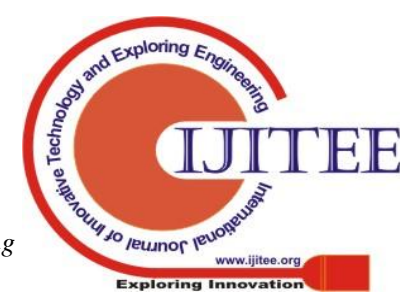


optimization algorithm will include and improve the better accuracy rate.

\section{CONCLUSION}

The aim of this research, reducing noise and improve the image quality using various filtering technique. I proposed the extension of adaptive bilateral method to apply the low frequency sub-bands of signal decomposed using a wavelet transform. The wavelet threshold is combined with adaptive bilateral filter. This technique is very efficient to eliminate noises in original noisy images and then to detected block boundary and texture regions discontinuities to adapt or control the parameters of spatial and intensity in bilateral method. The results show that MRI brain image de-noising using multi-resolution bilateral method is more effective pre-processing technique. The proposed segmentation technique uses novel strip method and the image is split into number of strips 3, 4, 5 and 6. A hybrid Assured Convergence PSO (ACPSO) and Fuzzy C-Mean Clustering (FCM) was proposed method. In segmentation algorithm presented in this paper gives $95 \%$ of accuracy rate to detect brain tissues when strip count is 4 . In future studies, various segmentation algorithm will be integrated and to improve the better accuracy rate.

\section{REFERENCES}

1. Emre Dand1l "A Computer-Aided Pipeline for Automatic Lung Cancer Classification on Computed Tomography Scans" Journal of Healthcare Engineering Volume 2018, Article ID 9409267, 12 pages

2. Lavanya M, Muthu Kannan P "Lung cancer segmentation and diagnosis of lung cancer staging using MEM (modified expectation maximization) algorithm and artificial neural network fuzzy inference system (ANFIS)" ISSN 0970-938X Biomed Research 201829 (14): 2919-2924.

3. Shraddha G. Kulkarni, Sahebrao B. Bagal "Lung Cancer Tumor Detection Using Image Processing And Soft Computing Techniques" International Journal of Science Technology and Management Volume 05 ISSN 2394-1537, May 2016

4. P. Kavitha, S. Prabakaran "Adaptive Bilateral Filter for Multi-Resolution in Brain Tumor Recognition" International Journal of Innovative Technology and Exploring Engineering (IJITEE) ISSN: 2278-3075, Volume-8 Issue-8 June, 2019.

5. P. Kavitha, S. Prabakaran "Designing a Feature Vector for Statistical Texture Analysis of Brain Tumor" International Journal of Engineering and Advanced Technology (IJEAT) ISSN: 2249-8958, Volume-8 Issue-5, June 2019.

6. P. Kavitha, S. Prabakaran "Brain Tumor Image Segmentation Using Hybrid Assured Convergence Particle Swarm Optimization and Fuzzy C-Means" International Journal of Scientific \& Technology Research Volume 7, Issue 8, August 2019.

7. P. Kavitha, S. Prabakaran "A Novel Hybrid Segmentation Method with Particle Swarm Optimization and Fuzzy C-Mean Based On Partitioning the Image for Detecting Lung Cancer" International Journal of Engineering and Advanced Technology (IJEAT) ISSN: 2249-8958, Volume-8 Issue-5, June 2019.

\section{AUTHORS PROFILE}

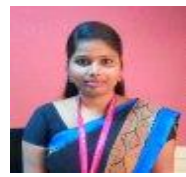

P Kavitha, Assistant Professor, Department of Information Technology, Bharath Institute of Higher Education and Research.

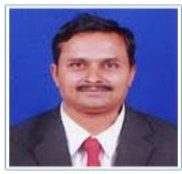

S.Prabakaran, Professor, Department of Computer Science and Engineering, SRM University 